\title{
Prognostic and clinicopathological significance of Beclin-I in non-small-cell lung cancer: a meta-analysis
}

This article was published in the following Dove Press journal: OncoTargets and Therapy

\author{
Tianliang Zhengl,* \\ Deping $\mathrm{Li}^{2}$,* \\ Zhanfeng $\mathrm{He}^{1}$ \\ Shuaibing Feng' \\ Song Zhao'
}

'Department of Thoracic Surgery,

The First Affiliated Hospital of

Zhengzhou University, ${ }^{2}$ Department of

Pain Management, Zhengzhou Hospital of Traditional Chinese Medicine,

Zhengzhou, China

*These authors contributed equally to this work
Correspondence: Song Zhao Department of Thoracic Surgery, The First Affiliated Hospital of Zhengzhou University, No. I Jianshe East Road, Zhengzhou 450052, China Tel +86 37I 6796 7I52

Email zzuzhaosong@yahoo.com
Background: Autophagy plays a key role in the development of non-small-cell lung cancer (NSCLC). Beclin-1 is essential for the initiation and regulation of autophagy. Accumulated studies have investigated the prognostic role of Beclin-1 in NSCLC, but conclusions remain controversial. Therefore, we conducted this meta-analysis to assess the potential significance of Beclin-1 in NSCLC.

Materials and methods: PubMed and Embase databases were searched for eligible studies published before December 31, 2017. Odds ratio (OR) was pooled to evaluate the clinicopathological significance of Beclin-1 in NSCLC. Hazard ratio (HR) was adopted to assess the association of Beclin-1 with overall survival (OS).

Results: Eight studies involving 1,159 patients were included in this meta-analysis. The pooled results showed that high Beclin-1 expression was significantly correlated with earlier tumor grade $(\mathrm{OR}=0.54,95 \% \mathrm{CI}$ : $0.36-0.81, P=0.003)$, less nodal involvement $(\mathrm{OR}=0.56,95 \% \mathrm{CI}$ : 0.37-0.86, $P=0.007)$, earlier TNM stage ( $\mathrm{OR}=0.62,95 \% \mathrm{CI}: 0.43-0.89, P=0.010)$, smaller tumor size $(\mathrm{OR}=0.54,95 \% \mathrm{CI}$ : $0.36-0.81, P=0.003)$, better differentiation $(\mathrm{OR}=0.48,95 \% \mathrm{CI}$ : $0.36-0.64, P<0.001)$, and less recurrence $(\mathrm{OR}=0.24,95 \% \mathrm{CI}: 0.14-0.41, P<0.001)$. Moreover, high level of Beclin-1 was significantly associated with better OS in NSCLC (HR $=0.41,95 \%$ CI: 0.26-0.64, $P<0.001)$.

Conclusion: Our meta-analysis suggests that high Beclin-1 expression predicts a better clinicopathological status and a better prognosis in NSCLC. Beclin-1 might act as a promising prognostic biomarker for NSCLC.

Keywords: non-small-cell lung cancer, autophagy, Beclin-1, prognosis, meta-analysis

\section{Introduction}

Lung cancer is the leading cause of cancer-related deaths in males, and the second leading cause in females worldwide. Non-small-cell lung cancer (NSCLC) accounts for $75 \%-80 \%$ of lung cancer patients. ${ }^{1}$ Multimodal therapeutic strategies including surgery, radiotherapy, chemotherapy, as well as molecular-targeted therapy have made great contributions to the treatment of NSCLC. However, owing to the rapid progression of tumors and late stage of diagnosis, the prognosis is dismal with a 5-year survival ranging between $7 \%$ and $18 \%$ in different countries. ${ }^{2}$ The clinical dilemma makes it urgent to develop more effective strategies for the diagnosis, treatment, and prognosis prediction of NSCLC. Previous researches mainly focused on apoptosis induced by various anticancer strategies. In recent years, a novel mechanism, autophagy, has received critical attention. 
Autophagy is an evolutionary conserved process in both prokaryotic and eukaryotic cells induced by metabolic stress or other stimuli. The basal level of autophagy is crucial for the survival of cells. Autophagy has a dual role in cancer cells, and may function as both a tumor suppressor and a tumor promoter in a context-dependent fashion. During carcinogenesis, autophagy may be regulated to match with the proliferation of cancer cells. In the early stage of carcinogenesis, autophagy may function as a tumor suppressor due to immunosurveillance, and the inhibition of autophagy may accelerate the progression of carcinogenesis, while autophagy may act as a tumor promoter in the late stage of tumor progression, which has to resist the hostile environment caused by hypoxia, nutrition deprivation, and metabolic stress., ${ }^{3,4}$ Moreover, the dynamic equilibrium of autophagy is critical to the survival of cancer cells, and the deregulation of autophagy may disturb the growth and differentiation of cancer cells. ${ }^{5}$ Some pharmaceuticals have the ability to resist cancer by regulating autophagy. For example, rapamycin enhances the anticancer effect of chemotherapy agents by upregulating autophagy, while chloroquine improves the anticancer effect of everolimus by inhibiting autophagy. ${ }^{6,7}$ Therefore, the modification of autophagy is considered a promising strategy for cancer therapy. A series of components called autophagy-related genes or proteins (Atg) are involved in the initiation and progression of autophagy. As the first identified mammalian Atg, Beclin-1 plays a key role in the initiation and regulation of autophagy. Beclin-1 is also the essential component of PI3K-III complex, which is composed of Vps34, Beclin-1, Vps15, and mAtg14. The interaction of Beclin-1 with Vps34 initiates autophagy by recruiting other Atgs to the phagophore. ${ }^{8}$ Moreover, Beclin-1 participates in the regulation of autophagy by binding with different molecules, such as UVRAG and Bcl-2 family proteins. ${ }^{9}$ The aberrant expression of Beclin-1 has been observed in a series of cancers, such as breast cancer, oral cancer, and gallbladder cancer. ${ }^{10-12}$ Numerous researches investigated the correlation between Beclin-1 and prognosis of NSCLC, but the conclusions were inconsistent. Therefore, the following meta-analysis was performed.

\section{Materials and methods Search strategy}

PubMed and Embase databases were searched for relevant studies that evaluated the prognostic and clinicopathological value of Beclin-1 in NSCLC published up to December 31, 2017. The search strategy included the following terms: "lung cancer" or "lung carcinoma" or "pulmonary cancer" or "pulmonary carcinoma" combined with "Beclin-1" or "BECN-1". Moreover, the references of relevant literatures were also reviewed manually for suitable studies.

\section{Inclusion and exclusion criteria}

Studies were primarily considered to be eligible if they satisfied all of these criteria: 1) patients were pathologically diagnosed with NSCLC; 2) the detection biomarker was Beclin-1; 3) sufficient data were supplied to assess the hazard ratios (HRs) and CIs of overall survival (OS), or adequate information could be extracted to estimate the correlation between Beclin-1 expression and clinicopathological characteristics; and 4) the language was limited to English. Furthermore, studies were excluded if they met one of the following criteria: 1) not written in English; 2) insufficient information to estimate the prognostic and clinicopathological correlation; and 3) duplicated data.

\section{Quality assessment}

Two investigators (D Li and S Feng) independently assessed the included studies in accordance with the NewcastleOttawa Quality Assessment Scale. ${ }^{13}$ This scale evaluated the quality of studies by awarding scores, and the total scores represented the quality of studies. A third investigator $(\mathrm{Z} \mathrm{He})$ checked the included studies for potential errors.

\section{Data extraction}

All data were extracted independently by two investigators (T Zheng and Z He). If there were disagreements that affected the quality of pooled results, a third investigator (S Zhao) assessed and determined the data independently. The following data were extracted: 1) general information including the name of the first author, publication year, country, number of patients, treatment strategy, follow-up period, and detection method of Beclin-1; 2) clinicopathological information including the correlation between Beclin-1 expression and tumor grade, nodal involvement, tumor node metastasis (TNM) stage, tumor size, tumor differentiation, and recurrence; and 3) HRs and 95\% CIs for OS were extracted from the studies if the data were supplied explicitly in the published studies. If the survival data were not reported, we extracted them from Kaplan-Meier curves for further calculation.

\section{Statistical analysis}

The patients were stratified into high-expression and lowexpression group according to the detection level of Beclin-1. Pooled OR and $95 \%$ CI were adopted to evaluate the correlation between Beclin-1 expression and clinicopathological 
characteristics, including gender, age, tumor grade, nodal involvement, TNM stage, tumor size, differentiation, and recurrence. Pooled HR and 95\% CI were calculated to estimate the correlation between Beclin-1 expression and OS. For studies that only provided the Kaplan-Meier curves, we estimated the HRs and 95\% CIs from the curves according to the method introduced by Parmar et al. ${ }^{14}$ The heterogeneity was assessed by the inconsistency index $I^{2}$, and was considered significant if $P$-value was $<0.1$ or $I^{2}$ was $>50 \%$. If the heterogeneity was not significant, the fixed-effect model was adopted; otherwise, the random-effect model was adopted. To estimate the reliability of the pooled result, sensitivity analysis was performed by omitting each single study. Publication bias was assessed by Begg's funnel plot and Egger's bias indicator test. ${ }^{15}$ Data were processed using Stata version 12.0 (Stata Corporation, College Station, TX, USA).

\section{Results}

\section{Search results and characteristics of eligible studies}

The flowchart of the literature search is shown in Figure 1. A total of 535 studies were identified according to the search strategy. Of these, 163 studies were removed because of duplication. By screening the abstracts, 324 studies were excluded due to irrelevant content or language limitation or because they were laboratory research reports or reviews. After screening the full text, 40 studies were excluded. Finally, eight studies were included for further analysis. $^{16-23}$
The general characteristics of the included studies are summarized in Table 1. The eight studies were published between 2011 and 2015. Five studies were conducted in China, ${ }^{16,20-23}$ two in Korea, ${ }^{17,18}$ and one in Greece. ${ }^{19}$ A total of 1,159 patients were included, and sample sizes for the included studies ranged between 79 and 262. The treatment strategy was surgery alone or multiple therapies, which comprised surgery, chemotherapy, and radiotherapy. The detection methods included immunohistochemistry and reverse transcription polymerase chain reaction. Four studies reported the HR data directly, ${ }^{17,20,21,23}$ and one study provided Kaplan-Meier curve. ${ }^{22}$ The quality scores ranged between 7 and 9 (Table 2).

\section{Correlation between Beclin-I expression and clinicopathological characteristics}

As shown in Figure 2 and Table 3, our meta-analysis showed that high expression of Beclin-1 was significantly correlated with earlier tumor grade $(\mathrm{OR}=0.54,95 \% \mathrm{CI}$ : $0.36-0.81$, $P=0.003$, fixed-effect model), less nodal involvement $(\mathrm{OR}=0.56,95 \% \mathrm{CI}: 0.37-0.86, P=0.007$, random-effect model $)$, earlier TNM stage $(\mathrm{OR}=0.62,95 \% \mathrm{CI}: 0.43-0.89$, $P=0.010$, random-effect model), smaller tumor size $(\mathrm{OR}=0.54$, 95\% CI: 0.36-0.81, $P=0.003$, fixed-effect model), better differentiation $(\mathrm{OR}=0.48,95 \% \mathrm{CI}: 0.36-0.64, P<0.001$, fixed-effect model), and less recurrence $(\mathrm{OR}=0.24,95 \% \mathrm{CI}$ : 0.14-0.41, $P<0.001$, fixed-effect model). On the contrary, the correlations were not statistically significant between Beclin-1 level and gender $(\mathrm{OR}=1.20,95 \% \mathrm{CI}$ : 0.69-2.07,

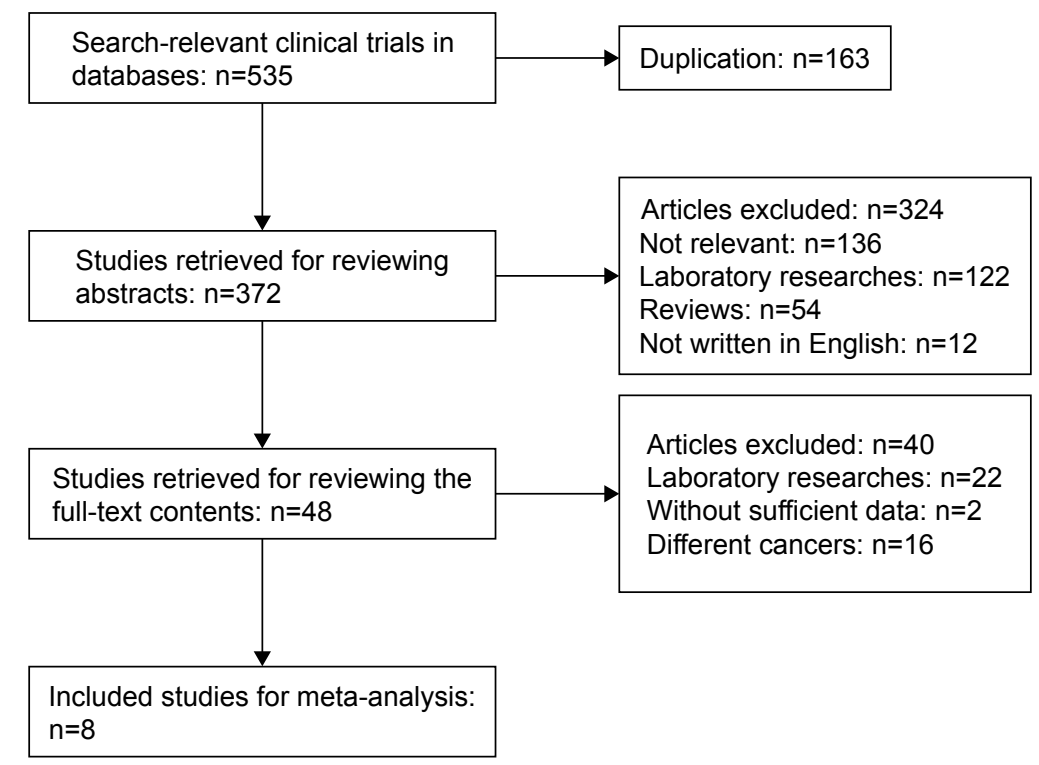

Figure I Flowchart of the literature search. 
Table I General characteristics of included studies

\begin{tabular}{|c|c|c|c|c|c|c|}
\hline Study & Year & Country & $\begin{array}{l}\text { Sample } \\
\text { size }\end{array}$ & Treatment & $\begin{array}{l}\text { Follow-up } \\
\text { (months) }\end{array}$ & Method \\
\hline Liu et $\mathrm{al}^{16}$ & 2011 & China & 79 & Surgery & NR & RT-PCR \\
\hline Kim et $\mathrm{al}^{17}$ & 2012 & Korea & 133 & Surgery & 32.5 & $\mathrm{IHC}$ \\
\hline Won et $\mathrm{al}^{18}$ & 2012 & Korea & 262 & Surgery & 65.1 & $\mathrm{IHC}$ \\
\hline Karpathiou et al ${ }^{19}$ & 2013 & Greece & 115 & Surgery & 32 & $\mathrm{IHC}$ \\
\hline Zhou et $\mathrm{al}^{20}$ & 2013 & China & 216 & Multiple & NR & $\mathrm{IHC}$ \\
\hline Jiang et $\mathrm{al}^{2 ।}$ & 2014 & China & 142 & Surgery & 31 & $\mathrm{IHC}$ \\
\hline Lv et $\mathrm{al}^{22}$ & 2015 & China & 108 & Surgery & 30 & $\mathrm{IHC}$ \\
\hline Wang et $\mathrm{al}^{23}$ & 2015 & China & 104 & Multiple & 48.5 & $\mathrm{IHC}$ \\
\hline
\end{tabular}

Abbreviations: NR, not recorded; RT-PCR, reverse transcription polymerase chain reaction; IHC, immunohistochemistry.

$P=0.525$, random-effect model $)$, and age $(\mathrm{OR}=1.00,95 \% \mathrm{CI}$ : $0.65-1.55, P=0.989$, random-effect model). These results indicated that NSCLC with high expression of Beclin-1 exhibited less aggressive clinicopathological behaviors.

\section{Correlation between Beclin-I expression and OS of NSCLC}

Five studies investigated the association between Beclin-1 expression and OS. ${ }^{17,20-23}$ A random-effect model was adopted because of significant heterogeneity among studies $\left(I^{2}=71.0 \%, P=0.008\right)$. As shown in Figure 3, the pooled result showed that high expression of Beclin-1 was significantly associated with better OS in NSCLC ( $\mathrm{HR}=0.41,95 \% \mathrm{CI}$ : 0.26-0.64, $P<0.001)$.

\section{Sensitivity analysis}

Sensitivity analysis was performed to determine the reliability of pooled result. The result showed that the pooled HR was not influenced by omitting each single study, indicating the consistency of the result (Figure 4).

\section{Publication bias}

The Begg's funnel plot and Egger's bias indicator test were introduced to assess the publication bias for OS. No publication bias was observed for OS (Begg's test: $P=0.327$; Egger's test: $P=0.061$ ) (Figure 5).

\section{Discussion}

Autophagy is essential for the development and prognosis of cancer. Current researches mainly focused on the prognostic role of autophagy-related markers Beclin-1 and LC3 in cancer. ${ }^{24,25}$ As an essential component, Beclin-1 participates in the initiation and regulation of autophagy. Moreover, Beclin-1 is also the key part of the PI3K-III complex, which is composed of PI3K-III-Beclin-1-Vps15-mAtg14. ${ }^{26}$ The conjugation of PI3K-III and Beclin-1 stimulates the synthesis of PIP3 and promotes the elongation of phagopore. Besides, the PI3K-III-Beclin-1 complex activates the recruitment of other Atgs to the phagopore, which initiates the formation and maturation of autophagosome. ${ }^{27}$ A series of autophagyrelated components regulate autophagy by binding to the functional domains of Beclin-1. For example, UVRAG and Barkor protein promote the maturation of phagopore and formation of autophagosome by binding to the specific functional domains of Beclin-1. Bcl-2 protein interacts with Beclin-1 and inhibits autophagy by suppressing the formation of PI3K-III-Beclin-1 complex. ${ }^{28}$ The aberrant expression of Beclin-1 was found in various cancers; for example, the loss or low expression of Beclin-1 was found in esophageal cancer, ${ }^{29}$ gastric cancer, ${ }^{30}$ and hepatocellular carcinoma, ${ }^{31}$ while the elevated expression of Beclin-1 was found in colorectal cancer, ${ }^{32}$ gallbladder cancer, ${ }^{33}$ and papillary thyroid carcinoma. ${ }^{34}$ The prognostic role of Beclin-1 was investigated

Table 2 Clinicopathological and prognostic characteristics of included studies

\begin{tabular}{|c|c|c|c|c|c|c|c|}
\hline Study & $\begin{array}{l}\text { T grade } \\
(1-2 / 3-4)\end{array}$ & $\begin{array}{l}\text { N grade } \\
(0 / 1-3)\end{array}$ & $\begin{array}{l}\text { TNM stage } \\
\text { (I-II/III-IV) }\end{array}$ & $\begin{array}{l}\text { Tumor size } \\
(<3 \mathrm{~cm} />3 \mathrm{~cm})\end{array}$ & $D(\mathbf{W}+\mathbf{M} / \mathbf{P})$ & $\begin{array}{l}\text { Source } \\
\text { of } H R\end{array}$ & $\begin{array}{l}\text { Quality } \\
\text { score }\end{array}$ \\
\hline Liu et al $^{16}$ & NR & $41 / 38$ & $46 / 33$ & $38 / 41$ & $51 / 28$ & None & 7 \\
\hline Kim et $\mathrm{al}^{17}$ & $96 / 37$ & $82 / 51$ & $93 / 40$ & $49 / 84$ & NR & Reported & 9 \\
\hline Won et $\mathrm{al}^{18}$ & $23 \mid / 31$ & $167 / 95$ & $195 / 67$ & $62 / 200$ & $220 / 42$ & None & 8 \\
\hline Karpathiou et al ${ }^{19}$ & $N R$ & $N R$ & $82 / 33$ & NR & $32 / 56$ & None & 8 \\
\hline Zhou et $\mathrm{al}^{20}$ & $160 / 56$ & $116 / 100$ & $138 / 78$ & NR & $116 / 100$ & Reported & 9 \\
\hline Jiang et $\mathrm{al}^{2 \mathrm{I}}$ & NR & $80 / 62$ & $107 / 35$ & NR & $85 / 57$ & Reported & 9 \\
\hline Lv et $\mathrm{al}^{22}$ & NR & $55 / 53$ & $86 / 22$ & NR & $62 / 46$ & Estimated & 7 \\
\hline Wang et $\mathrm{al}^{23}$ & NR & $58 / 46$ & $47 / 57$ & NR & $67 / 37$ & Reported & 8 \\
\hline
\end{tabular}

Abbreviations: T, tumor; N, node; TNM, tumor node metastasis; D, differentiation; $\mathrm{W}+\mathrm{M}$, well and moderate differentiation; P, poor differentiation; HR, hazard ratio; NR, not recorded. 
A

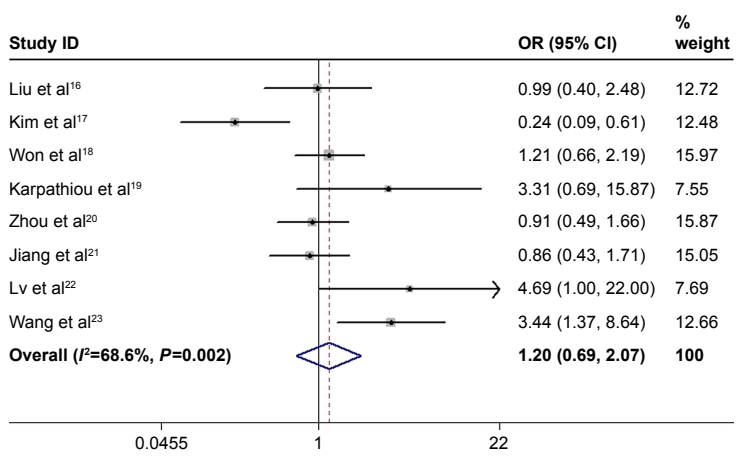

C

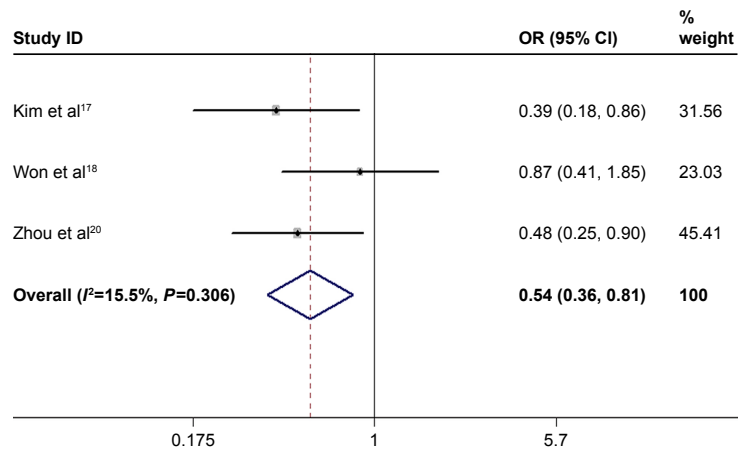

E

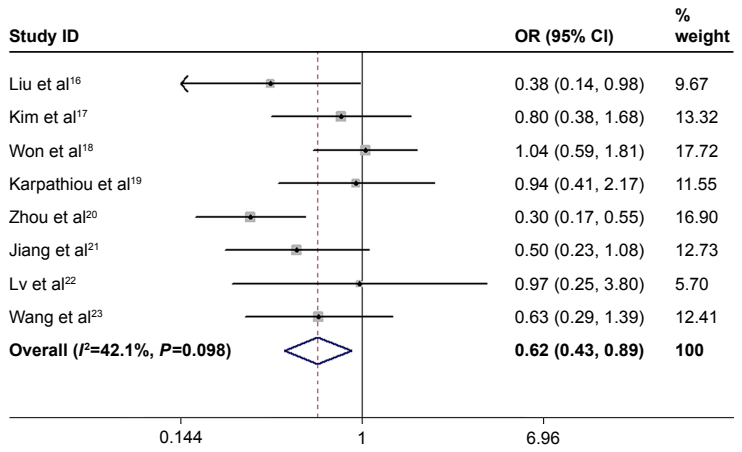

G

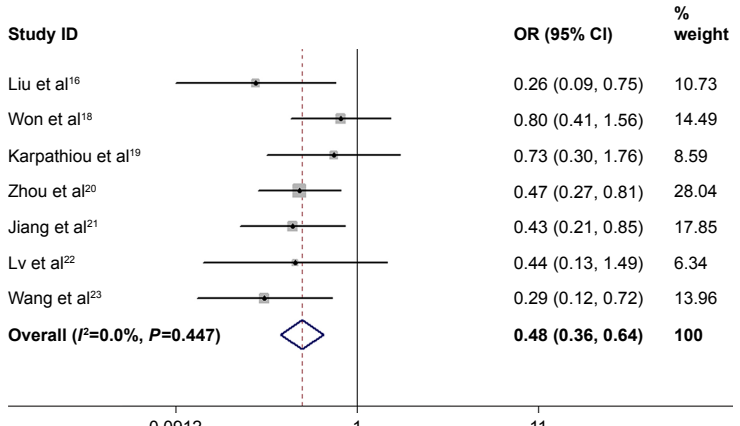

B

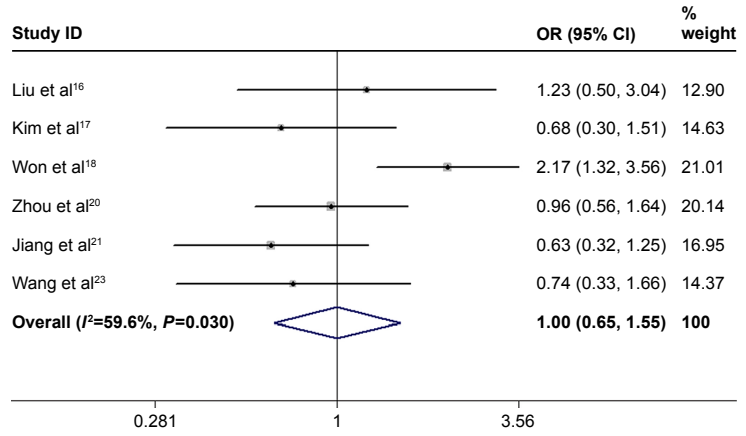

D

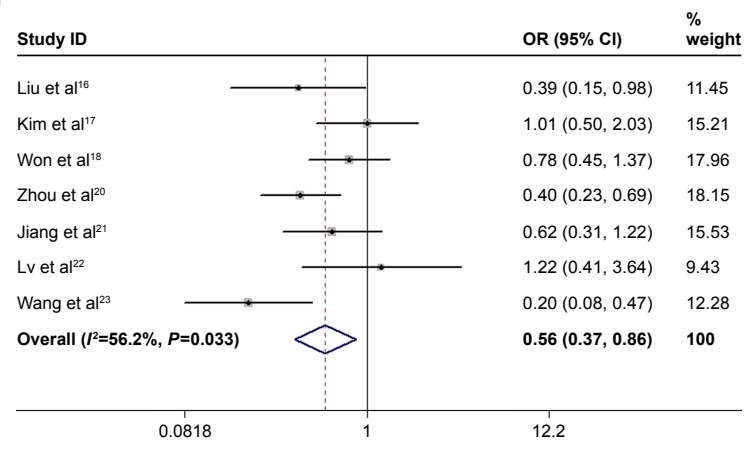

$\mathbf{F}$

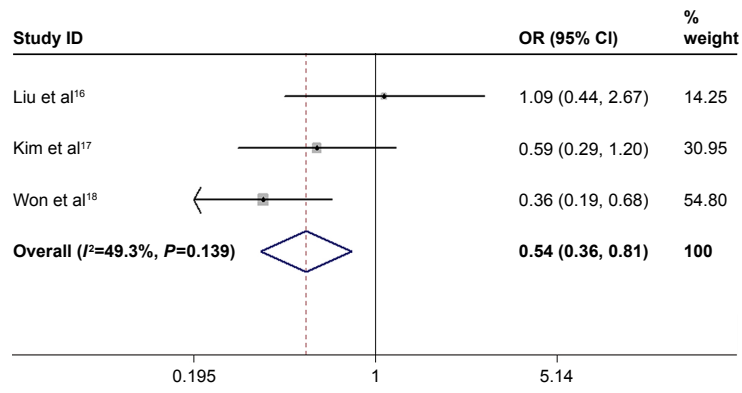

H

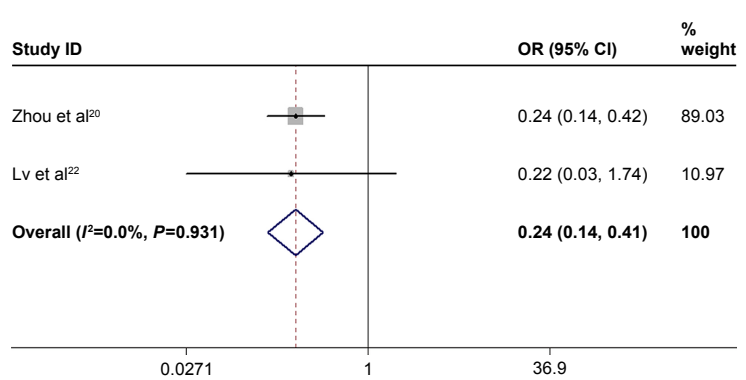

Figure 2 Forest plots for the relationship between Beclin-I and gender (A), age (B), T grade (C), N grade (D), TNM stage (E), tumor size (F), tumor differentiation $(\mathbf{G})$, and recurrence $(\mathbf{H})$ of NSCLC patients.

Note: Weights are from random effects analysis.

Abbreviations: T, tumor; N, node; TNM, tumor node metastasis; NSCLC, non-small-cell lung cancer; OR, odds ratio.

in various types of cancers, but the conclusions were inconsistent. It was shown by some studies that high level of Beclin-1 predicted a better outcome in colorectal carcinoma, ${ }^{35}$ oral carcinoma, and multiple myeloma, ${ }^{36,37}$ while other researchers showed that high level of Beclin-1 was correlated with a worse outcome in certain cancers such as endometrial carcinoma and nasopharyngeal carcinoma. ${ }^{38,39}$ Moreover, some researches showed that Beclin-1 was not associated with 
Table 3 Correlation analyses on Beclin-I and clinicopathological characteristics

\begin{tabular}{|c|c|c|c|c|c|c|}
\hline \multirow{2}{*}{$\begin{array}{l}\text { Clinicopathological } \\
\text { features }\end{array}$} & \multirow[t]{2}{*}{ Studies } & \multirow[t]{2}{*}{ Patients } & \multirow[t]{2}{*}{ OR $(95 \% \mathrm{Cl})$} & \multirow[t]{2}{*}{$P$-value } & \multicolumn{2}{|c|}{ Heterogeneity } \\
\hline & & & & & $I^{2}(\%)$ & $P$-value \\
\hline Gender (male vs female) & $8^{16-23}$ & 1,159 & $1.20(0.69,2.07)$ & 0.525 & 68.6 & 0.002 \\
\hline Age (old vs young) & $6^{16-18,20,21,23}$ & 936 & $1.00(0.65,1.55)$ & 0.989 & 59.6 & 0.030 \\
\hline T grade (T3-4 vs TI-2) & $3^{17,18,20}$ & 611 & $0.54(0.36,0.81)$ & 0.003 & 15.5 & 0.306 \\
\hline N grade (NI-3 vs N0) & $7^{16-18,20-23}$ & $\mathrm{I}, 044$ & $0.56(0.37,0.86)$ & 0.007 & 56.2 & 0.033 \\
\hline TNM (III-IV vs I-II) & $8^{16-23}$ & 1,159 & $0.62(0.43,0.89)$ & 0.010 & 42.1 & 0.098 \\
\hline Size $(>3 \mathrm{~cm}$ vs $<3 \mathrm{~cm})$ & $3^{16-18}$ & 474 & $0.54(0.36,0.81)$ & 0.003 & 49.3 & 0.139 \\
\hline $\mathrm{D}(\mathrm{P}$ vs $\mathrm{W}+\mathrm{M})$ & $7^{16,18-23}$ & 999 & $0.48(0.36,0.64)$ & 0.000 & 0.0 & 0.447 \\
\hline Recurrence (yes vs no) & $2^{20,22}$ & 324 & $0.24(0.14,0.4 I)$ & 0.000 & 0.0 & 0.931 \\
\hline
\end{tabular}

Abbreviations: $O R$, odds ratio; $D$, differentiation; $P$, poor differentiation; $W+M$, well and moderate differentiation.

prognosis of cancers. ${ }^{33,40}$ Even in the same type of cancer, the prognostic role of Beclin-1 was divergent. ${ }^{41,42}$ Some researches discussed the prognostic significance of Beclin-1 in NSCLC, but the conclusions were controversial. ${ }^{16,22}$ So, we conducted this meta-analysis to obtain definitive conclusions.

As far as we know, this is the first meta-analysis that systematically investigates the clinicopathological and prognostic role of Beclin-1 in NSCLC. Our research showed that high expression of Beclin-1 was associated with a better prognosis in NSCLC. Moreover, the correlation analysis showed that high level of Beclin-1 was significantly correlated with earlier tumor grade, lower nodal involvement, earlier TNM stage, smaller tumor size, better differentiation, and less recurrence.

The TNM system has made great contributions to therapy strategy selection and prognosis prediction of NSCLC.
However, the great variation in prognosis within the same TNM stage indicates that TNM system alone is far from satisfying the clinical requirement. A possible explanation is that TNM system estimates NSCLC based on anatomic information only, which ignores the biological and physiological information of NSCLC. However, the physiological status is essential for the development and metastasis of NSCLC. It is necessary to identify biomarkers that are associated with the biological characteristics to combine with TNM system for prognosis prediction. As one of the most important biomarkers, Beclin-1 is closely related to the activity of autophagy in NSCLC. Our research showed that high level of Beclin-1 was associated with earlier tumor grade, less nodal involvement, earlier TNM stage, smaller tumor size, better differentiation, and less recurrence. This suggested that Beclin-1 level was negatively correlated with progression and metastasis of NSCLC. This conclusion was consistent with

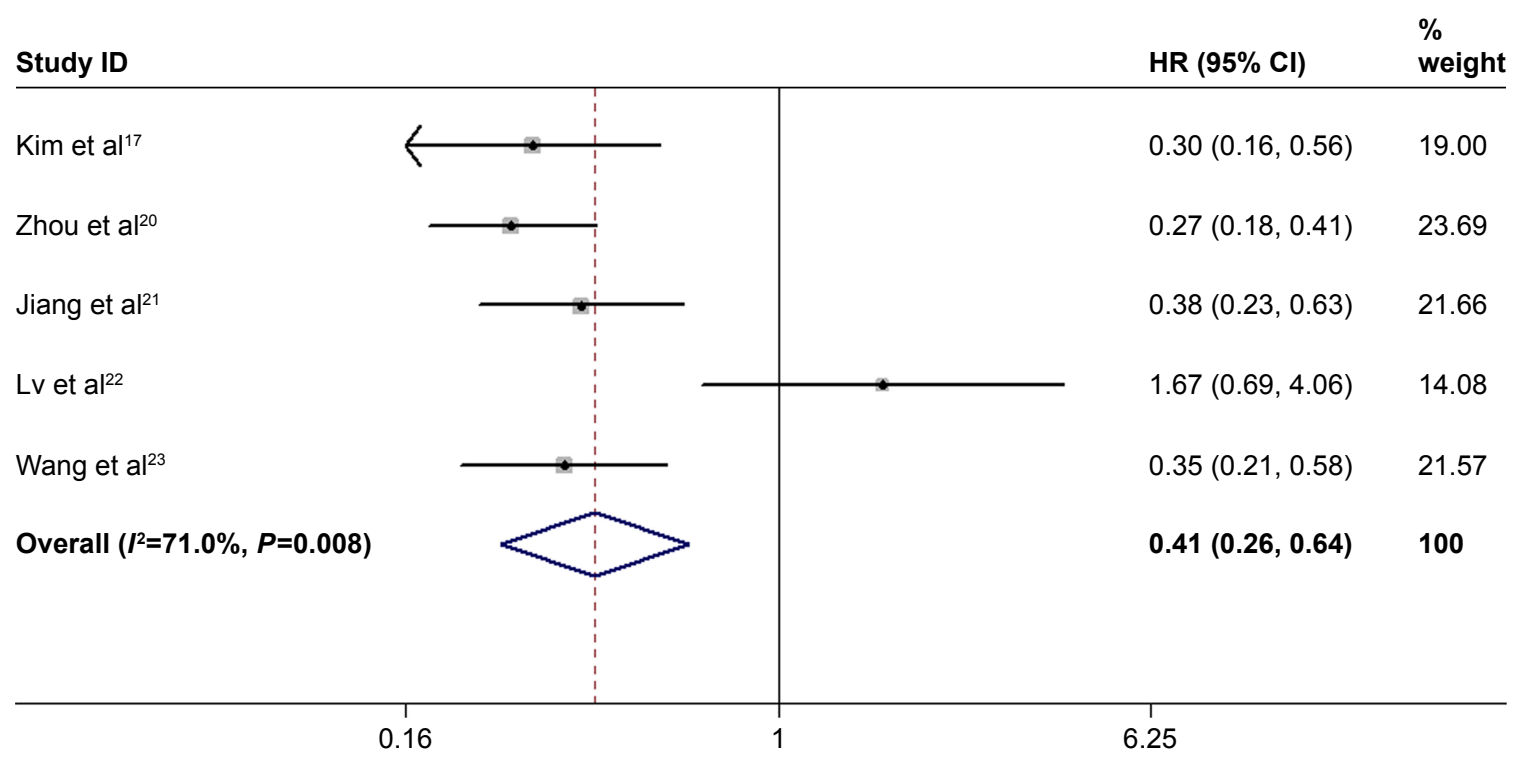

Figure 3 Forest plot for the association between Beclin-I and OS of NSCLC patients. Note: Weights are from random effects analysis.

Abbreviations: OS, overall survival; NSCLC, non-small-cell lung cancer; HR, hazard ratio. 
Meta-analysis random-effects estimates (exponential form) study omitted

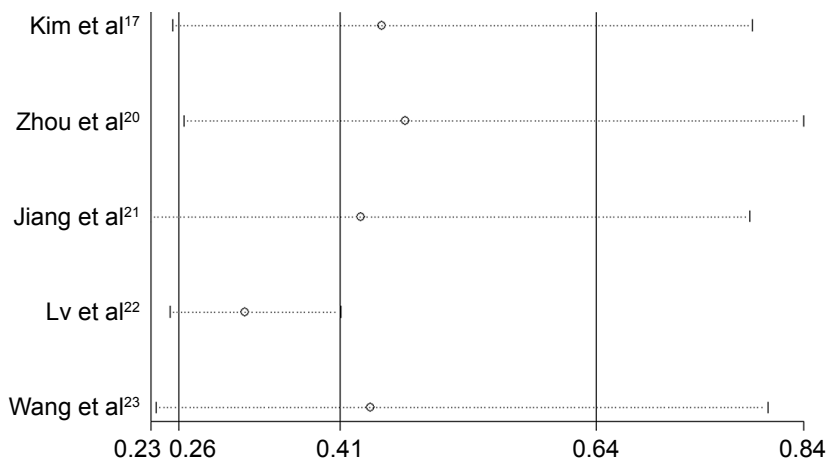

Figure 4 Sensitivity analysis of studies assessing OS Abbreviation: OS, overall survival.

previous researches in vitro, which showed that overexpression of Beclin-1 inhibited the proliferation and invasion of A549 human lung adenocarcinoma cells, while knockdown of Beclin-1 promoted the proliferation of A549 cells. ${ }^{43,44}$ Another meta-analysis investigated the clinicopathological significance of Beclin-1 in gastric cancer, and reached similar conclusions. In that meta-analysis, high level of Beclin-1 was associated with better differentiation and earlier TNM stage, but the level of Beclin-1 was not associated with lymph nodal involvement. ${ }^{45}$ The above results indicated that there was a reliable correlation between Beclin-1 expression and clinicopathological characteristics of cancer.

Five studies reported the correlation between Beclin-1 level and OS in NSCLC, and the pooled HR value was 0.41 (95\% CI: $0.26-0.64, P<0.001) .{ }^{17,20-23}$ This result indicated that high level of Beclin-1 predicted a significantly better OS, and Beclin-1 was a promising prognostic biomarker in
NSCLC. However, the prognostic significances of Beclin-1 in other cancers were inconsistent. A meta-analysis investigated the correlation between Beclin-1 and prognoses of gastric cancer, colorectal cancer, lymphoma, breast cancer, and lung cancer. The high level of Beclin-1 predicted a better OS in gastric cancer, lymphoma, breast cancer, and lung cancer, while Beclin-1 was not correlated with OS of colorectal cancer. ${ }^{46}$ Meanwhile, another meta-analysis explored the prognostic role of Beclin-1 in colorectal cancer, and the result showed that high level of Beclin-1 predicted a worse OS in colorectal cancer. ${ }^{47}$ The divergence between the two meta-analyses might be due to different inclusion criteria and different statistical indicators. In He et al's meta-analysis, ${ }^{46}$ HR was adopted to investigate the prognostic role of Beclin-1, while in Han's meta-analysis, ${ }^{47}$ relative risk was employed.

Although obvious heterogeneity was observed for OS ( $\left.I^{2}=71.0 \%, P=0.008\right)$, it was inappropriate to perform subgroup analyses due to the lack of included studies. The sensitivity analysis was conducted to determine the reliability of the pooled result. The combined HR was not influenced by omitting each single study, demonstrating the consistency of the result.

\section{Limitations}

There were some limitations that should not be ignored. First, the number of included studies and total sample size were limited. The subgroup analysis could be performed only if more studies were included. Second, the language was restricted to English, which might have excluded potentially eligible studies that were written in other languages. However, we tried to review the abstracts of the English versions of these studies, and confirmed that these studies were not qualified for our research. Third, there was no unified

\section{B Egger's publication bias plot}

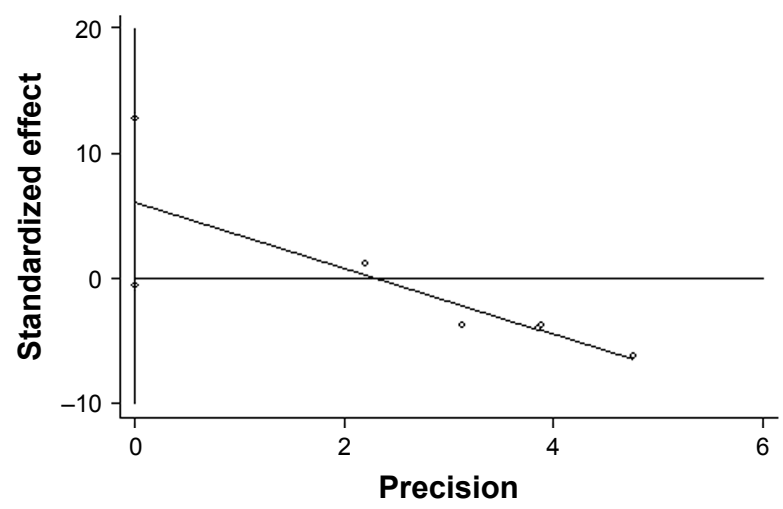

Figure 5 Begg's funnel plot (A) and Egger's bias indicator test (B) for publication bias of Beclin-I and OS. Abbreviations: OS, overall survival; HR, hazard ratio; SE, standard error. 
cut-off criterion for Beclin-1 to distinguish patients with high expression from patients with low expression. If there was a unified criterion, the heterogeneity among studies could be eliminated. Finally, there were no sufficient data to extract HR for disease-free survival (DFS). Although we extracted the information for recurrence rate, and estimated the correlation between Beclin-1 level and recurrence, the conclusion might be better if the combined HR for DFS could be calculated.

\section{Conclusion}

This meta-analysis showed that high Beclin-1 expression was correlated with earlier tumor grade, less nodal involvement, earlier TNM stage, smaller tumor size, better differentiation, and less recurrence of NSCLC. Moreover, high Beclin-1 expression predicted a better OS in NSCLC. Beclin-1 might act as a promising prognostic biomarker that can be used to improve decision making in the treatment of NSCLC.

\section{Acknowledgment}

This study was supported by the Youth Innovation Fund Project of the First Affiliated Hospital of Zhengzhou University.

\section{Disclosure}

The authors report no conflicts of interest in this work.

\section{References}

1. Torre LA, Siegel RL, Jemal A. Lung cancer statistics. Adv Exp Med Biol. 2016;893:1-19.

2. Coleman MP, Forman D, Bryant H, et al; ICBP Module 1 Working Group. Cancer survival in Australia, Canada, Denmark, Norway, Sweden, and the UK, 1995-2007 (the International Cancer Benchmarking Partnership): an analysis of population-based cancer registry data. Lancet. 2011;377(9760):127-138.

3. Rao S, Yang H, Penninger JM, Kroemer G. Autophagy in non-small cell lung carcinogenesis: a positive regulator of antitumor immunosurveillance. Autophagy. 2014;10(3):529-531.

4. Guo JY, White E. Autophagy, metabolism, and cancer. Cold Spring Harb Symp Quant Biol. 2016;81:73-78.

5. Mowers EE, Sharifi MN, Macleod KF. Autophagy in cancer metastasis. Oncogene. 2017;36(12):1619-1630.

6. Dai ZJ, Gao J, Ma XB, et al. Antitumor effects of rapamycin in pancreatic cancer cells by inducing apoptosis and autophagy. Int J Mol Sci. 2012;14(1):273-285.

7. Grimaldi A, Santini D, Zappavigna S, et al. Antagonistic effects of chloroquine on autophagy occurrence potentiate the anticancer effects of everolimus on renal cancer cells. Cancer Biol Ther. 2015;16(4): 567-579.

8. Itakura E, Mizushima N. Atg14 and UVRAG: mutually exclusive subunits of mammalian Beclin 1-PI3K complexes. Autophagy. 2009;5(4): 534-536.

9. McKnight NC, Zhenyu Y. Beclin 1, an essential component and master regulator of PI3K-III in health and disease. Curr Pathobiol Rep. 2013;1(4): 231-238.
10. Gu Y, Chen T, Li G, et al. Lower Beclin 1 downregulates HER2 expression to enhance tamoxifen sensitivity and predicts a favorable outcome for ER positive breast cancer. Oncotarget. 2017;8(32):52156-52177.

11. Park JY, Kim HS, Cho H, et al. Clinicopathologic correlation of autophagy-related Beclin-1 expression in gallbladder cancer. Hepatogastroenterology. 2014;61(134):1494-1500.

12. Liu JL, Chen FF, Chang SF, et al. Expression of Beclin family proteins is associated with tumor progression in oral cancer. PLoS One. 2015; 10(10): 0141308 .

13. Stang A. Critical evaluation of the Newcastle-Ottawa scale for the assessment of the quality of nonrandomized studies in meta-analyses. Eur J Epidemiol. 2010;25(9):603-605.

14. Parmar MK, Torri V, Stewart L. Extracting summary statistics to perform meta-analysis of the published literature for survival endpoints. Stat Med. 1998;17(24):2815-2834.

15. Egger M, Davey Smith G, Schneider M, Minder C. Bias in meta-analysis detected by a simple, graphical test. BMJ. 1997;315(7109):629-634.

16. Liu J, Lin Y, Yang H, Deng Q, Chen G, He J. The expression of $\mathrm{p} 33^{\mathrm{ING} 1}$, p53, and autophagy-related gene Beclin1 in patients with non-small cell lung cancer. Tumour Biol. 2011;32(6):1113-1121.

17. Kim KM, Yu TK, Chu HH, et al. Expression of ER stress and autophagyrelated molecules in human non-small cell lung cancer and premalignant lesions. Int J Cancer. 2012;131(4):E362-E370.

18. Won KY, Kim GY, Lim SJ, Kim YW. Decreased Beclin-1 expression is correlated with the growth of the primary tumor in patients with squamous cell carcinoma and adenocarcinoma of the lung. Hum Pathol. 2012;43(1):62-68.

19. Karpathiou G, Sivridis E, Koukourakis M, et al. Autophagy and Bcl-2/ BNIP3 death regulatory pathway in non-small cell lung carcinomas. APMIS. 2013;121(7):592-604.

20. Zhou W, Yue C, Deng J, et al. Autophagic protein beclin 1 serves as an independent positive prognostic biomarker for non-small cell lung cancer. PLoS One. 2013;8(11):e80338.

21. Jiang L, Liang X, Liu M, et al. Reduced expression of liver kinase B1 and Beclin1 is associated with the poor survival of patients with nonsmall cell lung cancer. Oncol Rep. 2014;32(5):1931-1938.

22. Lv ZQ, Han JJ, Liu YQ, et al. Expression of beclin 1 in non-small cell lung cancer: an immunohistochemical study. Clin Respir J. 2015;9(3): 359-365.

23. Wang X, Du Z, Li L, Shi M, Yu Y. Beclin 1 and p62 expression in nonsmall cell lung cancer: relation with malignant behaviors and clinical outcome. Int J Clin Exp Pathol. 2015;8(9):10644-10652.

24. Wang J, Pan XL, Ding LJ, Liu DY, Da-Peng L, Jin T. Aberrant expression of Beclin-1 and LC3 correlates with poor prognosis of human hypopharyngeal squamous cell carcinoma. PLoS One. 2013;8(7):e69038.

25. Valente G, Morani F, Nicotra G, et al. Expression and clinical significance of the autophagy proteins Beclin 1 and LC3 in ovarian cancer. Biomed Res Int. 2014;2014:462658.

26. Kang R, Zeh HJ, Lotze MT, Tang D. The Beclin 1 network regulates autophagy and apoptosis. Cell Death Differ. 2011;18(4):571-580.

27. Hoyer-Hansen M, Jaattela M. Connecting endoplasmic reticulum stress to autophagy by unfolded protein response and calcium. Cell Death Differ. 2007;14(9):1576-1582.

28. Toton E, Lisiak N, Sawicka P, Rybczynska M. Beclin-1 and its role as a target for anticancer therapy. $J$ Physiol Pharmacol. 2014;65(4): 459-467.

29. Weh KM, Howell AB, Kresty LA. Expression, modulation, and clinical correlates of the autophagy protein Beclin-1 in esophageal adenocarcinoma. Mol Carcinog. 2016;55(11):1876-1885.

30. Yu S, Li G, Wang Z, et al. Low expression of MAP1LC3B, associated with low Beclin-1, predicts lymph node metastasis and poor prognosis of gastric cancer. Tumour Biol. 2016;37(11):15007-15017.

31. Qiu DM, Wang GL, Chen L, et al. The expression of beclin-1, an autophagic gene, in hepatocellular carcinoma associated with clinical pathological and prognostic significance. BMC Cancer. 2014;14:327.

32. Guo GF, Jiang WQ, Zhang B, et al. Autophagy-related proteins Beclin-1 and LC3 predict cetuximab efficacy in advanced colorectal cancer. World J Gastroenterol. 2011;17(43):4779-4786. 
33. Chen Y, Yan J, Yu S, Wang X, Zheng Q. Over-expression of beclin-1 in gallbladder carcinoma and its relationship with prognosis. Contemp Oncol (Pozn). 2014;18(3):171-176.

34. Li X, Xu H, Ma H. Beclin 1 is highly expressed in papillary thyroid carcinoma and correlates with lymph node metastasis. Acta Chir Belg. 2013;113(3):175-181.

35. Yang Z, Ghoorun RA, Fan X, et al. High expression of Beclin-1 predicts favorable prognosis for patients with colorectal cancer. Clin Res Hepatol Gastroenterol. 2015;39(1):98-106.

36. Hu Z, Zhong Z, Huang S, et al. Decreased expression of Beclin1 is significantly associated with a poor prognosis in oral tongue squamous cell carcinoma. Mol Med Rep. 2016;14(2):1567-1573.

37. Jung G, Roh J, Lee H, et al. Autophagic markers BECLIN 1 and LC3 are associated with prognosis of multiple myeloma. Acta Haematol. 2015;134(1):17-24.

38. Giatromanolaki A, Koukourakis MI, Koutsopoulos A, Chloropoulou P, Liberis V, Sivridis E. High Beclin 1 expression defines a poor prognosis in endometrial adenocarcinomas. Gynecol Oncol. 2011;123(1): $147-151$.

39. Wan XB, Fan XJ, Chen MY, et al. Elevated Beclin 1 expression is correlated with HIF-1alpha in predicting poor prognosis of nasopharyngeal carcinoma. Autophagy. 2010;6(3):395-404.

40. Zhang MY, Gou WF, Zhao S, et al. Beclin 1 expression is closely linked to colorectal carcinogenesis and distant metastasis of colorectal carcinoma. Int J Mol Sci. 2014;15(8):14372-14385.
41. Geng QR, Xu DZ, He LJ, et al. Beclin-1 expression is a significant predictor of survival in patients with lymph node-positive gastric cancer. PLoS One. 2012;7(9):e45968.

42. Masuda GO, Yashiro M, Kitayama K, et al. Clinicopathological correlations of autophagy-related proteins LC3, Beclin 1 and p62 in gastric cancer. Anticancer Res. 2016;36(1):129-136.

43. Wang W, Fan H, Li X, et al. Beclin 1 promotes apoptosis and decreases invasion by upregulating the expression of ECRG4 in A549 human lung adenocarcinoma cells. Mol Med Rep. 2016;14(1):355-360.

44. Wang W, Fan H, Zhou Y, Duan P, Zhao G, Wu G. Knockdown of autophagy-related gene BECLIN1 promotes cell growth and inhibits apoptosis in the A549 human lung cancer cell line. Mol Med Rep. 2013; 7(5):1501-1505.

45. Xia P, Wang JJ, Zhao BB, Song CL. The role of beclin-1 expression in patients with gastric cancer: a meta-analysis. Tumour Biol. 2013;34(6): 3303-3307.

46. He Y, Zhao X, Subahan NR, Fan L, Gao J, Chen H. The prognostic value of autophagy-related markers beclin-1 and microtubule-associated protein light chain $3 \mathrm{~B}$ in cancers: a systematic review and meta-analysis. Tumour Biol. 2014;35(8):7317-7326.

47. Han Y, Xue XF, Shen HG, et al. Prognostic significance of Beclin-1 expression in colorectal cancer: a meta-analysis. Asian Pac J Cancer Prev. 2014;15(11):4583-4587.
OncoTargets and Therapy

\section{Publish your work in this journal}

OncoTargets and Therapy is an international, peer-reviewed, open access journal focusing on the pathological basis of all cancers, potential targets for therapy and treatment protocols employed to improve the management of cancer patients. The journal also focuses on the impact of management programs and new therapeutic agents and protocols on

\section{Dovepress}

patient perspectives such as quality of life, adherence and satisfaction. The manuscript management system is completely online and includes a very quick and fair peer-review system, which is all easy to use. Visit http://www.dovepress.com/testimonials.php to read real quotes from published authors. 